Chapter 5

\title{
Connexin 36 is a Negative Regulator of Differentiation in Human Neuroblastoma
}

\author{
Mandeep Sidhu and Daniel J. Belliveau \\ Additional information is available at the end of the chapter \\ http://dx.doi.org/10.5772/55643
}

\section{Introduction}

Neuroblastoma is the most common extracranial solid tumor to present in children [1]. Neuroblastoma arises from bipotential sympathoadrenal progenitor cells of the trunk neural crest. During normal development, sympathoadrenal progenitors differentiate to form the sympathetic ganglia and adrenal medulla, however in neuroblastoma they form tumors at these sites instead [1, 2].

Retinoic acid is now employed in multi-modal therapy to treat high-risk neuroblastoma and eradicate minimal residual disease [3]. All-trans retinoic acid (ATRA) diminishes MYCN oncogene expression, arrests proliferation, and induces differentiation of neuroblastoma cells $[4,5]$.

Gap junctional intercellular communication (GJIC) and connexins (Cx) have been implicated in carcinogenesis and differentiation. GJIC is often perturbed and connexins are typically downregulated or aberrantly localized in cancer cells, including IMR-32 neuroblastoma cells $[6,7]$. Many connexins have been identified as tumor suppressors when overexpressed in cancer cells [6]. For example, overexpression of $\mathrm{C} x 43$ resulted in growth suppression of communication deficient Neuro-2A murine neuroblastoma cells [8]. In addition, connexins have been shown to enhance the differentiation of cancer cell lines [8,9]. For example, overexpression of $\mathrm{C} \times 32$ and $\mathrm{C} \times 43$ resulted in enhanced nerve growth factor induced neurite outgrowth in PC12 cells [10].

Gap junctions are membrane channels that allow intercellular communication between adjacent cells [11]. GJIC involves the passage of ions, second messengers, and metabolites less than $1 \mathrm{kDa}$ in size between cells [11, 12]. Gap junctions and their constituent proteins, connexins, regulate cellular processes such as homeostasis, growth, and differentiation [6, 13]. Gap 
junctions are formed from the docking of two connexons or hemichannels contributed by adjacent cells. Each connexon in turn is comprised of a hexamer of connexin subunits [11]. There are 21 known members of the human connexin family, most with unique spatial and temporal expression patterns [14].

Cx36 is a recently identified connexin that is expressed in neurons and pancreatic beta cells [15-17]. Cx36 is highly expressed in the developing nervous system, however its expression is decreased or lost by the second post-natal week in many structures [18, 19]. In adults, Cx36 is the predominant connexin involved in electrical synapses [20].

The differentiating agent, retinoic acid, increases GJIC and Cx43 expression in multiple cell types [21-23]. Interestingly, the mouse $C x 36$ gene contains a retinoid $X$ receptor binding motif indicating that $C \times 36$ may be susceptible to transcriptional regulation by retinoic acid [24].

In this study, we explored the effect of the neuronal connexin, Cx36, on the differentiation of SH-SY5Y human neuroblastoma cells. SH-SY5Y cells are often utilized as a model system for studies of neural disease, as well as in studies of differentiation $[25,26]$. The effect of ATRA on Cx36 expression was first examined in SH-SY5Y cells. Furthermore, the effects of Cx36 on proliferation and features of differentiation including neuritogenesis and molecular differentiation marker expression were investigated by manipulating Cx36 expression.

\section{Experimental procedures}

\subsection{Cell culture}

The neuroblastoma cell line, SH-SY5Y was obtained from American Type Culture Collection. SH-SY5Y is a proliferative cell line with a documented doubling time of 48 hours. SH-SY5Y cells were maintained in Dulbecco's Modified Eagle Medium (DMEM, Invitrogen) with high glucose, and supplemented with $10 \%$ fetal bovine serum (FBS, HyClone), $100 \mathrm{U} / \mathrm{ml}$ penicillin and streptomycin (Invitrogen) and $2 \mathrm{mM} \mathrm{L-glutamine} \mathrm{(Invitrogen).} \mathrm{For} \mathrm{cell} \mathrm{passage,} \mathrm{cells} \mathrm{were}$ washed with Hank's Buffered Salt Solution (HBSS, Sigma), treated with $0.25 \%$ trypsin and incubated for 5 minutes at $37^{\circ} \mathrm{C}$ to disperse the cells. Five $\mathrm{ml}$ of growth medium was added to the cells, and they were resuspended in a $10 \mathrm{~cm}$ dish in fresh growth medium after pipetting several times.

\subsection{Generation of stable cell lines}

\subsubsection{Overexpression}

Cells were plated at a density of $1 \times 10^{6}$ cells per well in a 6-well dish. The following day, $4 \mu \mathrm{g}$ of Cx36myc-DDK (Origene) or EGFP pcDNA3.1 were diluted in OPTI-MEM (Invitrogen) and mixed with $2.5 \mu$ l of lipofectamine 2000 (Invitrogen) in OPTI-MEM. The DNA and lipofectamine were mixed and the resulting complexes added to the cells after 20 minutes, which were also in OPTI-MEM. The cells and complexes were incubated for 6 hours and then returned to normal growth medium. The following day, transfected cells were passaged onto $10 \mathrm{~cm}$ dishes. 
Kill curves were performed on wild-type cells to determine optimal antibiotic concentrations. For stable cell lines, $800 \mu \mathrm{g} / \mathrm{ml} \mathrm{G418}$ was added to the cells, and all viable cells were passaged to a $10 \mathrm{~cm}$ dish following two weeks of selection, and G418 was reduced to $200 \mu \mathrm{g} / \mathrm{ml}$. However, cells were treated with $800 \mu \mathrm{g} / \mathrm{ml} \mathrm{G418} \mathrm{monthly} \mathrm{to} \mathrm{ensure} \mathrm{purity} \mathrm{of} \mathrm{the} \mathrm{culture.}$

\subsubsection{Knockdown}

shRNA knockdown: Cells were plated at a density of $5 \times 10^{5}$ cells per well in a 6-well dish. The following day, $1 \mu \mathrm{g}$ of one of four distinct shRNAs targeting Cx36 or non-effective (scrambled) control plasmids co-expressing GFP (Origene) were diluted in OPTI-MEM and mixed with 1.5 $\mu \mathrm{l}$ of lipofectamine 2000 in OPTI-MEM. The cells were then maintained as per the overexpression protocol. For stable cell lines, $800 \mathrm{ng} / \mathrm{ml}$ puromycin was added to the cells, and all viable cells were passaged to a $10 \mathrm{~cm}$ dish following two weeks of selection, and puromycin was further increased to $1.5 \mu \mathrm{g} / \mathrm{ml}$. In addition, cells were treated with $2.5 \mu \mathrm{g} / \mathrm{ml}$ puromycin monthly to maintain purity of the culture due to the occasional appearance of non-fluorescent cells.

\subsection{Differentiation assay}

SHSY-5Y cells were plated on rat-tail collagen coated 6-well or $60 \mathrm{~mm}$ plates, at a density of 10000 cells $/ \mathrm{cm}^{2}$ in growth medium. The following day, cells were treated with $10 \mu \mathrm{M}$ of all trans-retinoic acid (Sigma) for 4 days, with medium and treatment refreshed every 2 days. Cells were then washed with HBSS and treated with $20 \mathrm{ng} / \mathrm{ml}$ of brain derived neurotrophic factor (BDNF) in serum-free DMEM for 6 days, with medium and BDNF replaced every 2 days (modified from [26]). Medium for transfected cells contained the appropriate antibiotic. RNA and protein were extracted at Days 0, 2, 4 and 10 of differentiation, and connexin and differentiation marker expression were analyzed. Images were taken at each timepoint with a Leica inverted epifluorescent microscope. The images were analyzed with the NeuronJ plugin for ImageJ $[27,28]$. For each image, neurites within the $0.16 \mathrm{~mm}^{2}$ field of view were traced. Neurites were classified as cell projections longer than one cell body length. For analysis of average neurite length, the mean length of all neurites in the field of view was computed. In order to calculate maximum neurite length, the length of the longest neurite in the field of view was analyzed. Finally, for neurite density, the number of neurites was divided by the number of cell bodies in the field of view.

\subsection{Real-time PCR}

RNA was extracted and purified using the Qiagen RNeasy mini kit, and $0.5 \mu \mathrm{g}$ of RNA was reverse transcribed using the qScript cDNA synthesis kit (Quanta Biosciences). Connexin primers were designed using Primer-Blast yielding the following sense and anti-sense sequences: Cx36, 5'-AAG GCA TCT CCC GCT TCT ACA - 3' and 5'- GCC AAC CAG GAA CCC AAT TT- $3^{\prime}$; Cx $45,5^{\prime}$-CTG GAG GCT CTG CAG CGG GA- $3^{\prime \prime}$ and $5^{\prime}$-TCT CCC GGG GAC CAT GAG GG- $3^{\prime}$; Cx43, 5'-GGT TAC ACT TGC AAA AGA GAT C- $3^{\prime}$ ' and 5'-GAG CAG CCA TTG AAA TAA GC- $3^{\prime}$. Differentiation markers: Neuropeptide Y (NPY), 5'- TCC AGC CCA GAG ACA CTG ATT-3' and 5'-AGG GTC TTC AAG CCG AGT TCT-3'; Growth associated 
protein 43 (Gap43), 5'-ACG ACC AAA AGA TTG AAC AAG ATG-3' and 5'-TCC ACG GAA GCT AGC CTG AA-3' (Origene). Adhesion molecule: Neural cell adhesion molecule (NCAM), 5' - CAT CAC CTG GAG GAC TTC TAC C-3' and 5' - CAG TGT ACT GGA TGC TCT TCA GG$3^{\prime}$ (Origene). Inhibitor of DNA Binding 2 (ID2), 5'-TTGTCAGCCTGCATCACCAGAG-3' and 5'-AGCCACACAGTGCTTTGCTGTC-3' (Origene). Nestin, 5'-TCAAGATGTCCCTCAGCCTGGA-3' and 5'-AAGCTGAGGGAAGTCTTGGAGC-3' (Origene). Values were normalized to those of the PPIA reference gene: cyclophillin A (PPIA), 5'-AGA CAA GGT CCC AAA GAC-3' and 5'ACC ACC CTG ACA CAT AAA-3'. All primer pairs were tested using standard curves with 10-fold serial dilutions, and selected only if the effiencies were within the $95-110 \%$ range over a minimum of 3 points. Three technical repeats were included for each biological replicate. The qPCR was performed in the CFX96 Real-Time PCR Detection System (Bio-Rad) using Perfecta Sybr Green Fastmix (Quanta Biosciences), and the data analyzed with CFX Manager software (Bio-Rad).

\subsection{Western blot analysis}

Protein was extracted using $18 \mathrm{mM}$ Tris, $123 \mathrm{mM} \mathrm{NaCl}, 10 \%$ glycerol, $1 \%$ NP40, and protease inhibitor cocktail (Calbiochem). Cell lysates were kept on ice for 5 minutes with frequent agitation, sonicated for 10 seconds, and lysates were cleared in a cold centrifuge at 10,000 rpm for 10 minutes. The supernatant was removed and used for western blotting. Protein concentration was measured using the BCA Protein Assay (Thermo Scientific). Thirty $\mu \mathrm{g}$ of protein were electrophoresed on $10 \%$ polyacrylamide gels and transferred to a nitrocellulose membrane. The membrane was blocked for one hour in 3\% BSA in PBS with 0.05\% Tween-20 (PBST), followed by incubation with primary antibodies: rabbit polyclonal Cx43 (Sigma) 1:2000 overnight at $4^{\circ} \mathrm{C}$; mouse monoclonal Gap43 (Invitrogen) 1:500 overnight at $4^{\circ} \mathrm{C}$; mouse monoclonal N-cadherin (BD) 1:2500 2 hours at room temperature; mouse monoclonal GAPDH (Millipore) 1:5000 for 2 hours at room temperature. Membranes were washed with PBST and incubated with secondary antibodies for one hour: horseradish peroxidase conjugated goat anti-mouse at a 1:5000 dilution (Thermo Scientific). Membranes were then washed with PBST and developed using enhanced chemiluminscence (Thermo Scientific). Zymed, Diatheva, Santa Cruz, and Sigma Cx36 antibodies were tested on blots with both positive and negative controls for $\mathrm{Cx} 36$. However, none of these antibodies were able to detect $\mathrm{C} \times 36$. Therefore, $\mathrm{Cx} 36$ expression was measured at the gene expression level. Protein expression was quantified with Quantity One software (Bio-Rad) by densitometric analysis. Protein levels were normalized to GAPDH.

\subsection{MTT proliferation assay}

Cells were plated at 20, 000 cells per well in a 48-well plate. At 0, 2, 4, and 6 days, $20 \mu$ l of MTT (Invitrogen) solution was added to each well and incubated for 4 hours. $200 \mu \mathrm{l}$ of SDS-HCl was then added to each well and incubated for 14 hours. The absorbance was measured at 570 $\mathrm{nm}$ on a $\mu$ Quant Biomolecular spectrophotometer (Bio-Tek). Increased absorbance indicates higher cell numbers. Four technical repeats were included in each biological replicate. 


\subsection{Statistical analysis}

Student's t-test was conducted for aggregate and neuritogenesis analysis between EGFP and Cx36 overexpressing cells. One-way analysis of variance (ANOVA) was conducted for the following experiments: gene expression analysis between wild-type cells, and neuritogenesis analysis between non-effective shRNA and Cx36 shRNAs. Tukey's multiple comparison posthoc test was used to assess differences in means between groups.

Two-way ANOVA was conducted for the following experiments: All gene and protein expression analysis and proliferation assays between transfected cells (overexpression and knockdown). Bonferroni's post-hoc test was conducted to assess differences in means between groups. $\mathrm{p}<0.05$ was considered significant for all tests. All statistical analysis was performed with GraphPad Prism 4.0 software.

\section{Results}

\subsection{Retinoic acid induces differentiation and upregulates Cx36 expression of SH-SY5Y neuroblastoma cells}

SH-SY5Y human neuroblastoma cells were induced to differentiate with all-trans retinoic acid to determine whether neuronal connexins are regulated by retinoic acid. Neuritogenesis, and Gap43 and NPY expression were assessed in order to evaluate the differentiation status of cells. Neuritogenesis is the process of morphological differentiation of neuronal cells whereby the cytoskeleton reorganizes and forms neurites, which are extensions of the cell body that serve as precursors of axons and dendrites [29]. Gap43 is found at high concentrations in the growth cone during neuritogenesis [30] and consequently it is often used as marker of neuronal differentiation [25,31]. NPY is a sympathetic peptide neurotransmitter and is also often used as a marker for differentiated neurons [31, 32].

SH-SY5Y cells were exposed to retinoic acid and BDNF treatment to induce cell differentiation. Neurite outgrowth was observed in treated cells beginning at day 2 (Figure 1A). Neuritogenesis continued through day 4 , and within 10 days extensive neurite networking was observed (Figure 1A). Untreated cells began to cluster and form aggregates by day 2, and continued to form large aggregates by day 10 (Figure 1A). Thus, retinoic acid and BDNF treatment induces the morphological differentiation of SH-SY5Y cells.

In order to determine whether retinoic acid regulates the expression of neuronal connexins, the effect of the differentiation treatment on $C x 36$ and $C x 45$ transcript expression was examined. Gap43 transcript expression was also assessed to confirm differentiation at the molecular level. RNA was isolated from untreated and treated cells at each timepoint, and real-time PCR was used to quantify gene expression. Messenger RNA for the growth cone marker, Gap43, was upregulated at days 4 and 10 of treatment compared to untreated cells (Figure 1B). Cx36 mRNA was increased in treated cells at 2, 4, and 10 days compared to untreated cells (Figure 1C). Cx45 mRNA, however, did not increase in treated cells (Figure 1D). These results indicate 
that transcription of the gene encoding $C x 36$ is upregulated by retinoic acid signaling, while Cx45 mRNA expression is unaffected.

\subsection{Cx36 suppresses neuritogenesis}

Cx36 mRNA was either stably overexpressed or knocked down in SH-SY5Y cells to determine the effect of CX36 on differentiation. SH-SY5Y cells were transfected and selected to stably overexpress Cx36 mRNA. Three weeks post-transfection, Cx36 overexpressing cells expressed 150 fold (141 +/- 27) more Cx36 mRNA than EGFP transfected cells (Figure 2A). Cx36 shRNA expressing cells exhibited an 88-99\% reduction in Cx36 mRNA levels in comparison to noneffective shRNA control prior to our differentiation studies (Figure 2B). Since all shRNA constructs coexpressed GFP, fluorescence was visualized to ensure continued expression of the construct during differentiation. The majority of differentiating cells (>85\%) continued to express all constructs during the ten day experiments (Figure 2C). Therefore, all constructs were successfully transfected and expressed by SH-SY5Y cells. In addition, Cx43 expression was analyzed following Cx36 overexpression and knockdown to ensure that Cx43 did not compensate for changes in Cx36 expression. Since Cx43 expression was not altered by changes in Cx36 expression, Cx43 does not appear to compensate for Cx36 in SH-SY5Y cells.

A
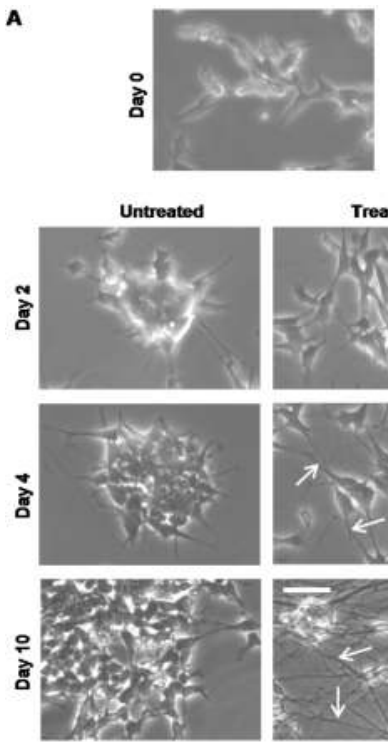

Treated
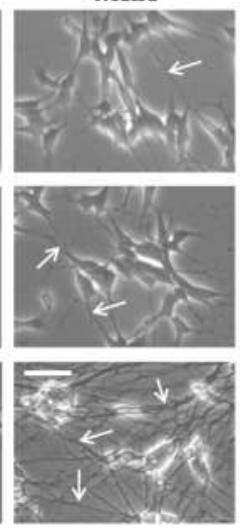
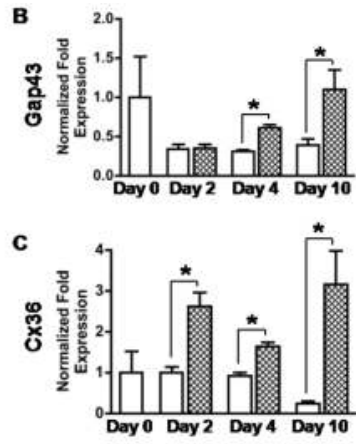

D

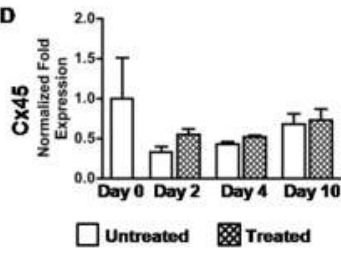

Figure 1. Retinoic acid induces differentiation of SH-SY5Y cells and upregulates Gap43 and Cx36 expression. Cells were seeded on rat-tail collagen coated dishes and treated with $10 \mu \mathrm{M}$ of retinoic acid the following day. During days $5-10$, cells were treated with $20 \mathrm{ng} / \mathrm{ml}$ of BDNF in serum-free medium. (A) Untreated cells formed increasingly larger aggregates over time. Neurite outgrowth (arrows) was observed beginning at days 2 and 4 of treatment, and dense neurite networks were formed following 10 days of treatment. Scale bar $=50 \mu \mathrm{m}$. (B-D) RNA was isolated from SH-SY5Y cells at days 0, 2, 4, and 10 of treatment. Real-time PCR was used to quantify mRNA expression. (B) Gap43 expression increased following 4 and 10 days of treatment. (C) Cx36 expression increased following 2, 4 and 10 days 
of treatment, as compared to untreated cells. (D) Cx45 expression did not increase in either treated or untreated cells. mRNA levels were normalized to PPIA. $\mathrm{N}=3$ (Day10, $\mathrm{N}=5$ ). ${ }^{*} \mathrm{p}<0.05$. Bars show mean $+/-\mathrm{S} . \mathrm{E}$.

Cx36 overexpressing cells were induced to differentiate in order to determine the effect of Cx36 on neuritogenesis. EGFP and Cx36 overexpressing cells were seeded for differentiation, and imaged at 0, 2, 4, and 10 days following differentiation treatment. EGFP expressing cells showed intense neurite networking by day 10 of differentiation treatment, while Cx36 overexpression diminished neuritogenesis (Figure 3A). There was no significant difference between EGFP and Cx36 overexpressing cells in average neurite length (Figure 3B). However, Cx36 overexpressing cells had significantly reduced neurite density compared to EGFP expressing cells (Figure 3C). Thus, our findings indicate that Cx36 downregulates retinoic acid induced neuritogenesis.

A

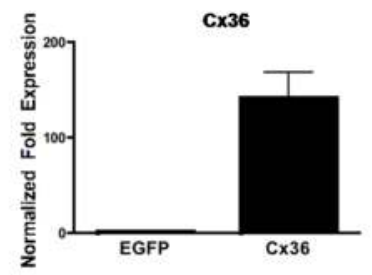

B
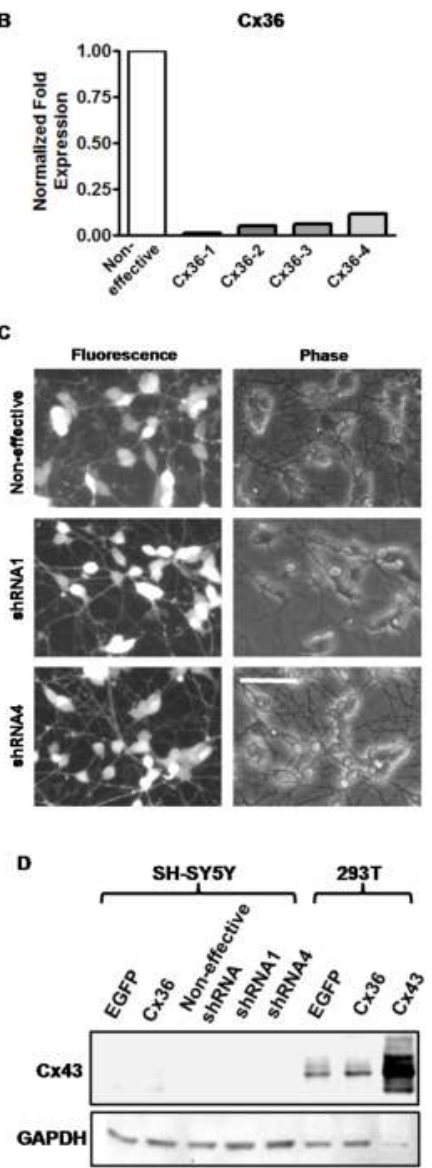

Figure 2. Cx36 overexpression and knockdown are maintained throughout differentiation. (A) SH-SY5Y cells were transfected and selected to stably overexpress CX36 or EGFP as a transfection control. RNA was isolated from transfected cells over the 10 day differentiation treatment, and Cx36 overexpressing cells showed at least 150 fold 
greater Cx36 expression levels than the EGFP control. (B, C) In addition, cells were transfected and selected to express four distinct Cx36 shRNA's and a non-effective shRNA transfection control, all of which coexpressed GFP. (B) RNA was isolated to determine expression levels prior to seeding cells for experiments. Cx36 shRNA expressing cells had decreased Cx36 transcript levels compared to non-effective control. RNA expression levels were normalized to PPIA mRNA. $N=3$ ( $B, N=1)$. ${ }^{*} p<0.05$. Bars show mean + /-S.E. (C) Most of the cell population expressed GFP, and thus the shRNA construct through ten days of differentiation treatment. Scale bar $=50 \mu \mathrm{m}$. (D) Cx43 expression was not detected and did not change following Cx36 overexpression or knockdown in SH-SY5Y cells.
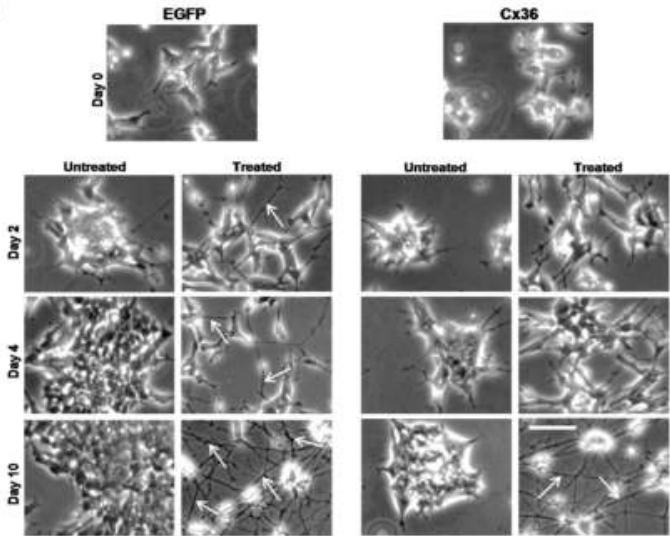

B

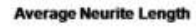

c

Neurite Density
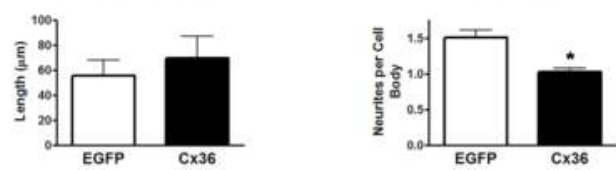

D
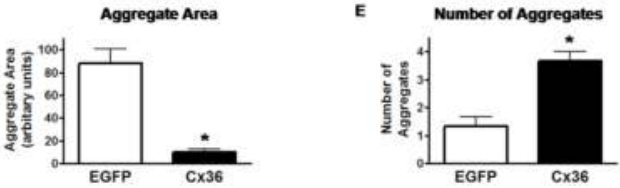

Figure 3. Cx36 overexpression diminishes neurite density and increases cell clumping in SH-SY5Y cells. Stably transfected EGFP and Cx36 overexpressing SH-SY5Y cells were treated with retinoic acid and BDNF. (A) EGFP expressing cells formed denser neurite networks (arrows) than Cx36 overexpressing cells following treatment. Untreated Cx36 overexpressing cells formed numerous small cell clumps whereas EGFP expressing cells formed larger aggregates. Scale Bar $=50 \mu \mathrm{m}$. (B,C) Treated cells were imaged at day 10 and neurite length and density were measured using Neuron J. (B) There was no significant difference in average neurite length between EGFP control and Cx36 overexpressing cells. (C) However, neurite density was diminished in Cx36 overexpressing cells. (D,E) Aggregate area and number were measured for untreated cells at day 10 using Image J. (D) Cx36 overexpressing cells formed aggregates with significantly smaller area, (E) however developed more numerous aggregates per field of view than EGFP expressing cells. * $p<0.05$. Bars show mean + /- S.E

To further demonstrate that Cx36 negatively regulates neuritogenesis, the effect of Cx36 knockdown on neuritogenesis was determined. An identical experimental design as the overexpression study was conducted between non-effective shRNA and Cx36 shRNA expressing cells. In these experiments, Cx36 shRNA expressing cells showed enhanced 
neuritogenesis (Figure 4A). There was no significant difference in average neurite length between Cx36 shRNA expressing and non-effective shRNA expressing cells (Figure 4B). However, neurite density was significantly increased in Cx36 shRNA expressing cells compared to non-effective shRNA expressing cells (Figure 4C). Therefore, knockdown of Cx36 enhanced retinoic acid induced neuritogenesis. These findings confirm that Cx36 diminishes retinoic acid induced neuritogenesis of SH-SY5Y cells.
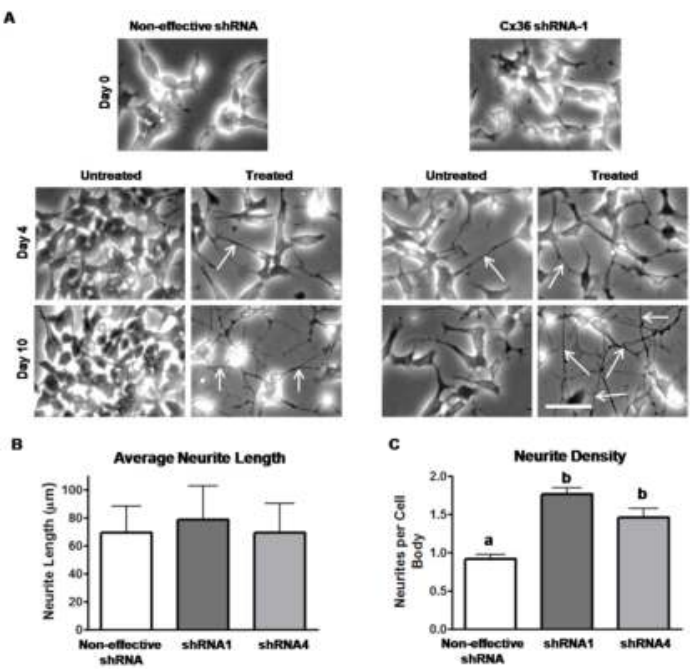

Figure 4. Knockdown of Cx36 increases neurite density and suppresses aggregate formation in SH-SY5Y cells. Cx36 shRNA expressing cells were plated and subjected to differentiation treatment over 10 days. (A) Treated Cx36 shRNA expressing cells exhibit enhanced neuritogenesis compared to non-effective control. Untreated non-effective shRNA expressing cells aggregate on collagen, while Cx36 shRNA expressing cells remain dispersed. Scale bar $=50 \mu \mathrm{m}$. (B,C) Differentiating cells were imaged at day 10 and neurite length and density were measured using Neuron J software. (B) There was no significant difference in average neurite length between non-effective shRNA expressing control and Cx36 shRNA expressing cells. (C) However, neurite density was higher in Cx36 shRNA expressing cells than in control. $\mathrm{N}=3$. Different letters indicate statistical significance. $\mathrm{p}<0.05$. Bars show mean $+/-\mathrm{S}$. E.

\subsection{Cx36 suppresses differentiation marker expression}

In order to determine whether Cx36 also negatively regulates other indicators of differentiation, the effect of Cx36 on the expression of molecular differentiation markers, Gap43 and NPY, was assessed. Transcript expression of the Gap43 differentiation marker was significantly reduced in Cx36 overexpressing cells at days 2 and 4 of treatment in comparison with EGFP expressing cells (Figure 5A). NPY mRNA levels were also significantly decreased in Cx36 overexpressing cells at days 2 and 10 of treatment compared to EGFP expressing cells (Figure 5B). Gap43 protein expression significantly increased upon differentiation treatment in EGFP expressing cells, however Cx36 overexpressing cells failed to significantly increase Gap43 expression following treatment (Figure 5 C \& D). Thus, Cx36 overexpression diminished the retinoic acid induced expression of molecular differentiation markers. 
A

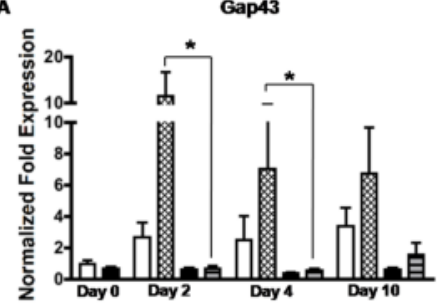

c

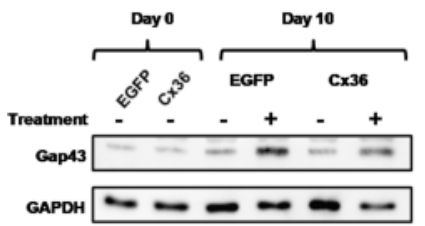

B

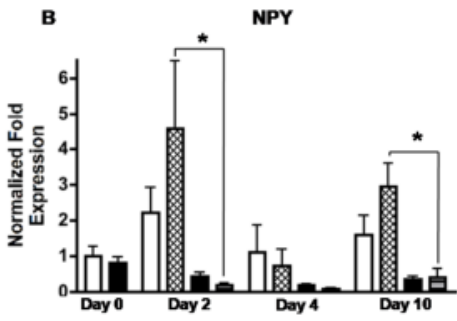

Gap43

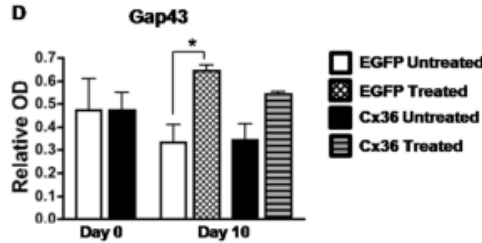

Figure 5. Cx36 overexpression diminishes retinoic acid induced Gap43 and NPY expression. (A,B) RNA was isolated from cells at day $0,2,4$, and 10 of differentiation treatment, and real-time PCR was used to quantify changes in mRNA expression. (C,D) Protein was extracted from cells at 0 and 10 days of treatment and western blot analysis was used to quantify protein expression. (A) Gap43 expression was diminished in Cx36 overexpressing cells at days 2 and 4 of treatment. (B) NPY expression is diminished at days 2 and 10 of treatment in Cx36 overexpressing cells. (C,D) Gap43 protein expression did not significantly differ between Cx36 overexpressing and EGFP expressing cells. Gap43 expression significantly increased upon treatment in EGFP cells, however it failed to significantly increase in treated Cx36 overexpressing cells. mRNA levels were normalized to PPIA and protein levels to GAPDH. $N=3 .{ }^{*} p<0.05$. Bars show mean +/- S.E.

The impact of Cx36 knockdown on expression of differentiation markers was assessed to confirm the effect of Cx36 on differentiation. Non-effective shRNA and Cx36 shRNA expressing cells were subjected to the 10 day differentiation treatment, and RNA was isolated at days 0, 4, and 10. Cx36 shRNA expressing cells exhibited significantly increased levels of Gap43 mRNA expression at day 10 of treatment compared with non-effective shRNA expressing cells (Figure 6A). In addition, Cx36 shRNA expressing cells had significantly higher NPY mRNA expression at day 0 and day 10 of differentiation treatment compared to non-effective shRNA expressing cells (Figure 6B). Untreated and treated Cx36 knockdown cells also expressed higher levels of Gap43 protein (Figure 6 C \& D). Furthermore, untreated Cx36 shRNA expressing cells expressed as much Gap43 protein as treated non-effective control, while treated Cx36 shRNA expressing cells expressed the highest levels of Gap43. Our findings show that Cx36 knockdown enhances retinoic acid induced differentiation marker expression.

\subsection{Cx36 promotes cell clumping}

An unexpected finding of our study was that Cx36 overexpression affected the adhesion and aggregation properties of cells on collagen coated dishes. Untreated EGFP and Cx36 overexpressing cells were plated on collagen coated plates for ten days. EGFP expressing cells behaved like wild-type cells, where they formed large cell aggregates over time, while Cx36 overexpressing cells formed cell clumps that were susceptible to lifting off the plate (Figure 3A). Cx36 overexpressing cells formed significantly smaller aggregates than EGFP expressing 


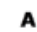

$\mathbf{A}$

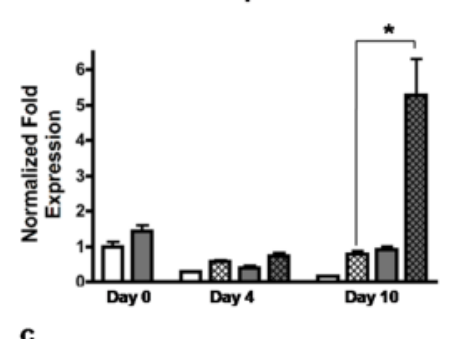

。

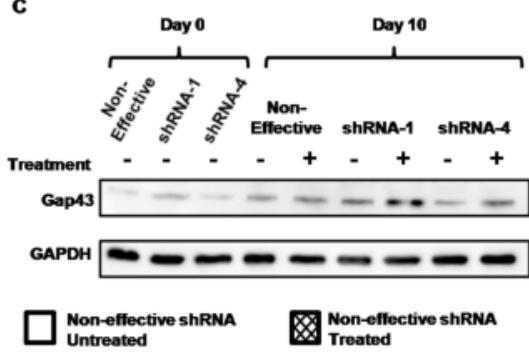

B

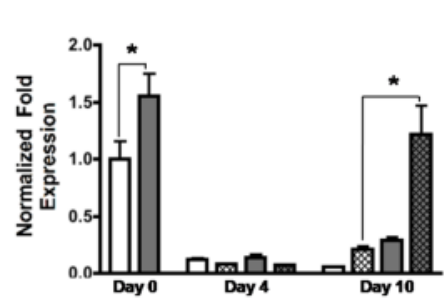

D

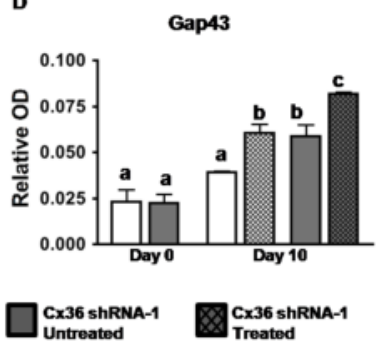

Figure 6. Cx36 knockdown enhances retinoic acid induced Gap43 expression and upregulates NPY expression. RNA and protein were isolated from cells at days 0,4 , and 10 of differentiation treatment. Real-time PCR and western blotting were used to quantify mRNA and protein expression respectively. (A) Gap43 expression is increased at day 10, (B) while NPY expression is increased at 0 and 10 days of treatment in Cx36 shRNA expressing cells compared to noneffective control. (C,D) Gap43 protein expression is enhanced in untreated and untreated Cx36 shRNA expressing cells compared to non-effective control at day 10 of treatment. mRNA levels were normalized to PPIA and protein normalized to GAPDH. N=3. Asterisks and different letters denote a statistically significant difference. $p<0.05$. Bars show mean +/-S.E.

cells (Figure 3D) in addition to significantly higher numbers of cell clumps than EGFP expressing cells (Figure 3E). Expression levels of two distinct cell adhesion molecules, NCAM and $\mathrm{N}$-cadherin, were examined to determine whether altered expression of these proteins mediated the cell clumping in Cx36 overexpressing cells. However, neither NCAM mRNA nor $\mathrm{N}$-cadherin protein levels showed any significant changes in Cx36 overexpressing cells during differentiation or in their untreated state (Figure 7). Therefore, Cx36 overexpression resulted in increased formation of small cell clumps, although the adhesion molecules involved in this process have not yet been identified.

In order to further explore the effect of Cx36 on cell clumping, the effect of Cx36 knockdown on aggregate formation was assessed in a comparable study between non-effective shRNA and Cx36 shRNA expressing SH-SY5Y cells. The untreated non-effective shRNA expressing cells formed large aggregates over ten days, similar to wild-type cells (Figure 4A). However, Cx36 shRNA expressing cells did not form aggregates, and maintained a dispersed phenotype (Figure 4A). NCAM expression was significantly higher in treated non-effective shRNA expressing cells than all other conditions (Figure 8A). N-cadherin expression did not meaningfully change between constructs (Figure 8 B \& C). Therefore, Cx36 knockdown prevents aggregate and cell clump formation in SH-SY5Y cells. However, the molecules through which Cx36 exerts its effect on cell aggregation have not yet been determined. 
A

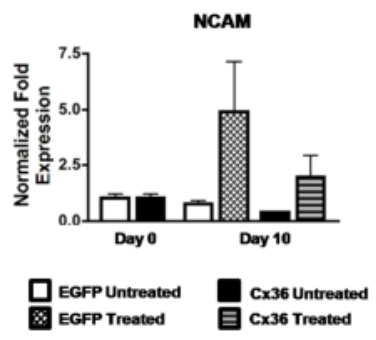

B

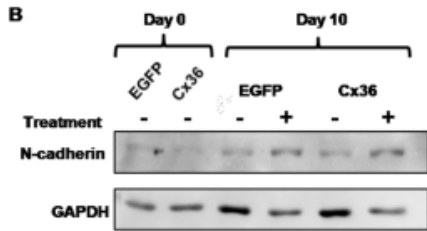

$\mathbf{C}$

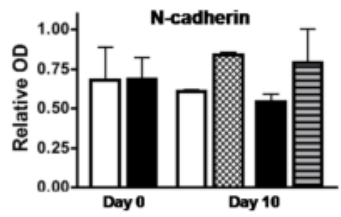

Figure 7. NCAM and $\mathrm{N}$-cadherin expression were not altered in differentiating Cx36 overexpressing cells. RNA and protein were isolated from cells at day 0 and 10 of treatment, and real-time PCR was used to quantify mRNA expression and western blotting to quantify protein expression. (A) NCAM mRNA expression levels were not significantly different between any condition. $(\mathbf{B}, \mathbf{C}) \mathrm{N}$-cadherin protein expression levels did not change between differentiation treatment or overexpression conditions ( $B$ \& $C$ ). mRNA levels were normalized to PPIA mRNA and protein levels to GAPDH. N=3. Bars show mean +/- S.E.

A
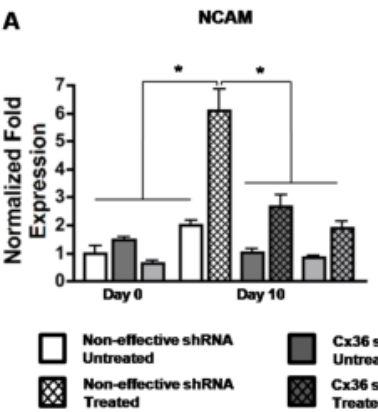

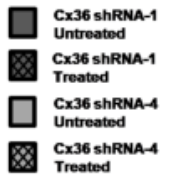

B

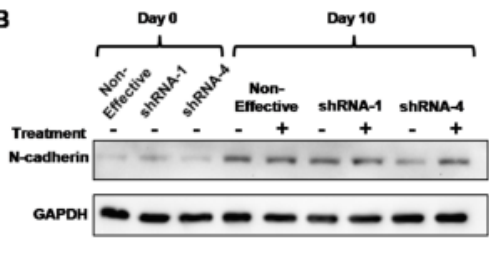

C

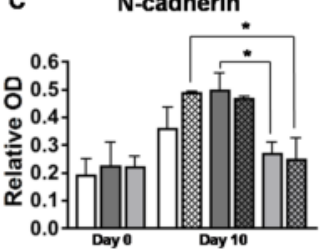

Figure 8. NCAM expression was reduced in Cx36 shRNA expressing cells and N-cadherin expression was only decreased in shRNA-4 expressing cells. RNA and protein were isolated from cells at day 0 and 10 of differentiation treatment, and real-time PCR was used to quantify mRNA expression and western blots to quantify protein expression. (A) NCAM mRNA expression was higher in treated non-effective control cells than all other conditions. (B,C) Ncadherin protein expression levels were decreased in shRNA-4 but not shRNA-1 expressing cells in comparison to non-effective control. mRNA levels were normalized to PPIA mRNA and protein levels to GAPDH. * $p<0.05$. $N=3$. Bars show mean $+/$ - S.E.

\subsection{Cx36 increases SH-SY5Y cell proliferation}

Neurons typically exit the cell cycle in order to terminally differentiate. Thus, cell proliferation was assessed in Cx36 overexpressing and knockdown cells to determine if changes in proliferation rate are a consequence of Cx36 manipulation of differentiation. The proliferation rates 
of EGFP and Cx36 overexpressing cells were assessed using the MTT proliferation assay. Absorbance was measured at 0,2, 4, and 6 days in culture. At day 6, Cx36 overexpressing cells had significantly higher absorbance than EGFP expressing cells implying enhanced cell proliferation in these cells (Figure 9A).

The identical assay was performed for non-effective shRNA and Cx36 shRNA expressing cells to confirm that Cx36 stimulates cell proliferation. At day 6, Cx36 shRNA expressing cells had significantly reduced absorbance in comparison to non-effective shRNA expressing cells (Figure 9B). Therefore, our findings indicate that Cx36 promotes proliferation of SH-SY5Y neuroblastoma cells.
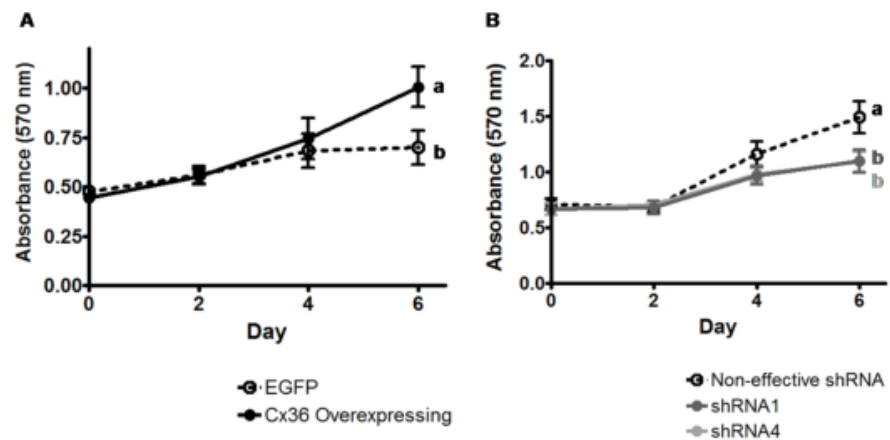

Figure 9. Cx36 overexpression increases SH-SY5Y cell proliferation while knockdown of Cx36 suppresses cell proliferation. Cells were seeded at 20,000 cells per well in 48-well plates and the MTT proliferation assay was conducted according to manufacturer's guidelines. (A) Following 6 days in culture, Cx36 overexpressing cells had a significantly higher proliferation rate than EGFP control cells. In contrast, Cx36 shRNA expressing cells had a significantly lower rate of proliferation than non-effective control. Different letters indicate significant difference. $\mathrm{p}<0.05$. $\mathrm{N}=3$. Points show mean + /-S.E.

Since high proliferation and lack of differentiation are traits of stem cells, the effect of Cx36 overexpression and knockdown on stem cell marker expression was assessed. ID2 and Nestin mRNA expression were upregulated following Cx36 overexpression (Figure 10 A \& B), but did not change upon Cx36 knockdown (Figure $10 \mathrm{C} \& \mathrm{D}$ ). These findings suggest that Cx36 overexpression may cause SH-SY5Y cells to adopt a stem-cell like phenotype.

\section{Discussion}

\subsection{Retinoic acid induces upregulation of Cx36}

Retinoic acid is often used as a therapeutic agent in the treatment of neuroblastoma to induce differentiation. In current neuroblastoma therapy, patients are given 13-cis retinoic acid during remission following treatment with chemotherapeutics to eradicate minimal residual disease $[3,33]$. Retinoic acid has been shown to upregulate Cx43 expression [21, 22], however there have been no reports of its effect on the neuronal connexins, CX36 and Cx45. The SH-SY5Y human 
neuroblastoma cell line is known to undergo extensive differentiation in response to retinoic acid treatment $[4,25]$. Therefore, the effect of retinoic acid on these neuronal connexins was investigated in theSH-SY5Y neuroblastoma cell line. We also investigated the IMR32 neuroblastoma cell line and while it expressed Cx36 and was responsive to retinoic acid, it had limited differentiation potential, consistent with previous findings by others [34]. Our study indicates that $C \times 36$ may be a target of retinoic acid signaling, since it is upregulated upon retinoic acid induced differentiation and $C x 36$ contains a retinoid $X$ receptor binding motif similar to that of the $C x 43$ gene [24]. Conversely, $C x 45$ does not appear to be regulated by retinoic acid. In fact, the influence of retinoic acid in either of its configurations, cis-retinoic acid or all-trans retinoic acid appears to have similar effects on connexins in a particular cellular context, however that effect can be very different depending on cellular environment, with examples of enhanced expression on Cx32 protein in LNCaP prostate cancer cells [35] versus suppression of Cx26 or 43 mediated coupling in squamous cell carcinoma cells [36]. Thus, we have identified $C x 36$ as a novel potential transcriptional target in retinoic acid signaling. In addition, since $C x 36$ expression increased upon retinoic acid induced differentiation, $C \times 36$ gene expression was manipulated to ascertain its effects on the differentiation and proliferation of SH-SY5Y cells.

A

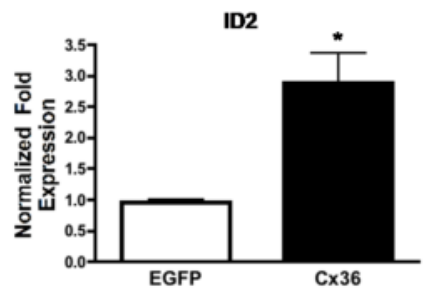

C

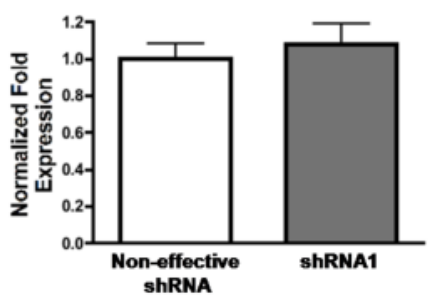

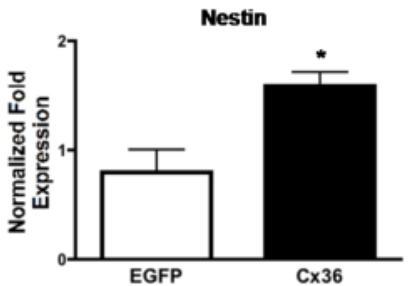

D

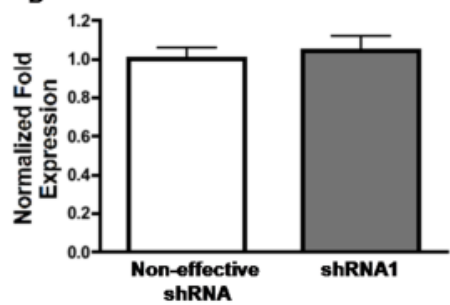

Figure 10. Cx36 overexpression results in increased stem cell marker expression, while Cx36 knockdown has no effect. RNA was isolated from cells and real-time PCR was used to quantify mRNA expression. (A,B) ID2 and Nestin expression increased following Cx36 overexpression. (C,D) However, ID2 and Nestin were unaffected by knockdown of $\mathrm{C} \times 36$. mRNA levels were normalized to PPIA. $\mathrm{N}=3$. Asterisks denote a statistically significant difference. $\mathrm{p}<0.05$. Bars show mean + /- S.E.

\subsection{Cx36 negatively regulates SH-SY5Y cell differentiation}

Cx36 appears to negatively regulate differentiation of SH-SY5Y human neuroblastoma cells. Accordingly, overexpression of Cx36 dimishes differentiation while knockdown of Cx36 
enhances differentiation. This appears to be in contradiction with the upregulation of Cx36 upon retinoic acid induced differentiation. However, since we were unable to measure protein expression, it is possible that although $C x 36 \mathrm{mRNA}$ is upregulated in response to retinoic acid, Cx36 protein expression is unaffected. Conversely, this discrepancy may also be due to the requirement of an optimal level of Cx36 for proper differentiation. Accordingly, the small increase in Cx36 expression induced by retinoic acid in wild-type cells does not appear to interfere with differentiation. However, since Cx36 overexpressing cells exhibit diminished differentiation and Cx36 knockdown enhances differentiation, it is apparent that substantial changes in $\mathrm{Cx} 36$ expression alter the process of differentiation.

The effect of Cx36 on SH-SY5Y differentiation is in contrast with what Hartfield et al (2011) have recently shown on Cx36 and neuronal progenitor cells [37]. Progenitors isolated from the rat hippocampus and striatum and grown in neurospheres showed increased differentiation with Cx36 overexpression, and diminished differentiation with Cx36 knockdown [37]. Although neuroblastoma arises from progenitor cells, these cancer cells likely respond differently to Cx36 levels than healthy progenitors. It is also possible that neuronal cells of the peripheral nervous system react differently to Cx36 than those of the central nervous system. The effect of $\mathrm{Cx} 36$ on differentiation is also in contrast with other connexins, which are known to enhance differentiation $[8,9]$. For example, overexpression of Cx43 and Cx32 enhanced nerve growth factor induced differentiation of PC12 cells [10]. Although connexin overexpression usually enhances differentiation, Todorova et al (2008) have shown that GJIC and Cx43 are required to maintain pluripotency of embryonic stem cells. Inhibition of GJIC or Cx43 knockdown resulted in reduced proliferation and stem cell marker expression, with a concomitant increase in differentiation marker expression [38]. Thus, in certain environments, as with our Cx36 overexpression study, connexin expression may be required to maintain the stem cell or cancer phenotype. This is further demonstrated in human breast cancer where gap junctions and GJIC are usually associated with tumor suppression however in aggressive breast cancers they may facilitate disease progression [39]. Our finding of Cx36 knockdown enhancing differentiation in fact parallels the natural expression patterns of Cx36, where it is highly expressed in many neural structures of the developing fetus but is subsequently downregulated during postnatal differentiation and maturation of the nervous system [18, 19]. This downregulation may be due in part to replacement of electrical synapses with chemical synapses [40], or it may indicate that Cx36 is actually detrimental in the process of terminal differentiation of certain tissues or cell types, as was shown in this study.

\subsubsection{Cx36 promotes clumping of SH-SY5Y cells}

A surprising finding was that altering Cx36 expression dramatically changed the adhesion and aggregation properties of SH-SY5Y cells. CX36 overexpression led to the formation of small cell clumps susceptible to lifting off the dish and growing in suspension. This type of growth is similar to that of neurospheres, which are suspended clusters of neural stem cells [41]. In fact, it is characteristic of the neuroblast subpopulations of SH-SY5Y cells to aggregate, lift off, and grow in suspension [42]. This neuroblastic phenotype appears to be stimulated by overexpression of $\mathrm{C} \times 36$, which is in accordance with our findings of Cx36 induced downre- 
gulation of differentiation. Interestingly, knockdown of Cx36 resulted in a dispersed cell phenotype.

NCAM and N-cadherin expression were assessed as potential mediators of the differential aggregate formation in Cx36 overexpressing and knockdown cells. Cx43 is known to colocalize with $\mathrm{N}$-cadherin and regulate its localization at the cell surface [43]. Although there have been no documented reports of Cx36 and N-cadherin interaction, we investigated whether manipulation of $\mathrm{C} x 36$ expression resulted in changes to $\mathrm{N}$-cadherin expression. In addition, we explored the effect of Cx36 expression on NCAM mRNA expression, as NCAM is upregulated in poorly differentiated and aggressive neuroblastomas [44]. Based on our studies, N-cadherin and NCAM are unlikely to be the primary adhesion molecule involved in this process. Therefore, while Cx36 expression has a dramatic effect on cell aggregation, the adhesion molecules mediating this process are yet to be identified.

\subsubsection{Cx36 increases cell proliferation}

There is a substantial linkage between connexin expression and cell growth and proliferation [6]. Our findings of increased cell proliferation with Cx36 overexpression, and diminished cell proliferation following knockdown of $\mathrm{Cx} 36$ are in contrast to other connexins, which typically act as tumor suppressors [6, 8]. However, in certain environments, connexins and gap junctional intercellular communication are required to maintain the proliferative ability of cells. For example, extravillous trophoblasts lost the ability to proliferate when GJIC was inhibited by a gap junction blocker or Cx40 antisense cDNA [45].

Additionally, Cx36 has been implicated in retinal cell survival following injury [46]. In light of the role of Cx36 in cell survival and its high expression during development when neural precursors are proliferating, it is not surprising to find that Cx36 serves to increase proliferation. It is also important to consider that Cx36 is regulated in a different manner from other connexins. For example, $\mathrm{C} \times 36$ channels show differential $\mathrm{pH}$ gating and experience very low sensitivity to voltage gating $[47,48]$. This $\mathrm{pH}$ gating may be important under pathological conditions such as ischemia, where Cx36 expression has been shown to be upregulated and involved in cell survival [49]. Therefore, the differential regulation mechanisms of Cx36 channels may suggest a different role for Cx36 in key cellular processes from that of other connexins.

In relation to differentiation, as neurons do not retain a capacity to divide, cells must exit the cell cycle and stop proliferating in order to terminally differentiate into neurons. Retinoic acid also exerts part of its differentiation inducing effects by inhibiting cell proliferation [50]. Therefore, the enhanced differentiation observed in Cx36 shRNA expressing cells may be partially due to their decreased proliferative ability.

SH-SY5Y cells overexpressing Cx36 adopt several characteristics of stem cells, including aggregation, high proliferation, and lack of differentiation. To determine whether these cells were also more stem cell like at the molecular level, the expression levels of ID2 and Nestin were assessed. ID2 is expressed in neural crest cells and is also known to increase proliferation and inhibit differentiation of neuroblastoma cells [51]. Nestin is often used as a neural stem 
cell marker as it is highly expressed in progenitor cells during development, but is downregulated in adults [52]. The upregulation of both ID2 and Nestin upon Cx36 overexpression suggests that increased levels of CX36 push SH-SY5Y cells towards a stem cell phenotype.

This study is the first to determine the effects of Cx36 in a cancer cell context. We have identified Cx36 as a novel potential transcriptional target of retinoic acid signaling. However, further research is required to determine whether $C x 36$ is a direct target of retinoic acid signaling. In addition, CX36 negatively regulates SH-SY5Y differentiation, which is in contrast to the effects of other connexins on differentiation. Furthermore, it appears that Cx36 promotes a neural stem cell like phenotype based on the diminished differentiation, enhanced clumping and increased proliferation in overexpressing cells, and the reversal of this phenotype in cells where Cx36 is knocked down.

However, it is unknown whether Cx36 mediates these changes in a gap junctional intercellular communication dependent manner or through interaction of Cx36 with binding partners, and thus downstream signaling pathways. Therefore, further research is required to elucidate the mechanisms underlying Cx36 mediated regulation of differentiation.

\section{Acknowledgements}

Supported by the National Sciences and Engineering Research Council of Canada to DJB.

\section{Author details}

Mandeep Sidhu and Daniel J. Belliveau*

*Address all correspondence to: dbellive@uwo.ca

Department of Anatomy and Cell Biology, Schulich of Medicine \& Dentistry and School of Health Studies, Faculty of Health Sciences, Western University, London, Ontario, Canada

\section{References}

[1] Brodeur, G. M. Neuroblastoma: biological insights into a clinical enigma. Nature reviewsCancer. (2003). Mar;PubMed PMID: PMID: 12612655; nrc1014 [pii]., 3(3), 203-16.

[2] Huber, K. The sympathoadrenal cell lineage: specification, diversification, and new perspectives. Developmental biology. (2006). Oct 15;PubMed PMID: PMID: 16928368; S0012-1606(06)00992-4 [pii]., 298(2), 335-43. 
[3] Wagner, L. M, \& Danks, M. K. New therapeutic targets for the treatment of high-risk neuroblastoma. Journal of cellular biochemistry. (2009). May 1;PubMed PMID: PMID: 19277986., 107(1), 46-57.

[4] Thiele, C. J, Reynolds, C. P, \& Israel, M. A. Decreased expression of N-myc precedes retinoic acid-induced morphological differentiation of human neuroblastoma. Nature. (1985). Jan 31-Feb 6;PubMed PMID: PMID: 3855502., 313(6001), 404-6.

[5] Sidell, N, Altman, A, Haussler, M. R, \& Seeger, R. C. Effects of retinoic acid (RA) on the growth and phenotypic expression of several human neuroblastoma cell lines. Experimental Cell Research. (1983). Oct;PubMed PMID: 6313408. Epub 1983/10/01. eng., 148(1), 21-30.

[6] Naus, C. C, \& Laird, D. W. Implications and challenges of connexin connections to cancer. Nature Reviews Cancer. (2010). Jun;PubMed PMID: 20495577. Epub 2010/05/25. eng., 10(6), 435-41.

[7] Arnold, J. M, Phipps, M. W, Chen, J, \& Phipps, J. Cellular sublocalization of Cx43 and the establishment of functional coupling in IMR-32 neuroblastoma cells. Molecular carcinogenesis. (2005). Mar;PubMed PMID: PMID: 15605363., 42(3), 159-69.

[8] Mao, A. J, Bechberger, J, Lidington, D, Galipeau, J, Laird, D. W, \& Naus, C. C. Neuronal differentiation and growth control of neuro-2a cells after retroviral gene delivery of connexin43. The Journal of biological chemistry. (2000). Nov 3;PubMed PMID: PMID: 10924505; M003917200 [pii]., 275(44), 34407-14.

[9] Banks, E. A, Yu, X. S, Shi, Q, \& Jiang, J. X. Promotion of lens epithelial-fiber differentiation by the C-terminus of connexin 45.6 a role independent of gap junction communication. Journal of cell science. (2007). Oct 15;PubMed PMID: PMID: 17895360; jcs.000935 [pii]., 120(20), 3602-12.

[10] Belliveau, D. J, Bani-yaghoub, M, Mcgirr, B, Naus, C. C, \& Rushlow, W. J. Enhanced neurite outgrowth in PC12 cells mediated by connexin hemichannels and ATP. The Journal of biological chemistry. (2006). Jul 28;PubMed PMID: PMID: 16731531; M600026200 [pii]., 281(30), 20920-31.

[11] Laird, D. W. The life cycle of a connexin: gap junction formation, removal, and degradation. Journal of Bioenergetics and Biomembranes. (1996). Aug;PubMed PMID: 8844328. Epub 1996/08/01. eng., 28(4), 311-8.

[12] Saez, J. C, Connor, J. A, Spray, D. C, \& Bennett, M. V. Hepatocyte gap junctions are permeable to the second messenger, inositol 1,4,5-trisphosphate, and to calcium ions. Proceedings of the National Academy of Sciences of the United States of America. (1989). Apr;PubMed PMID: 2784857. Epub 1989/04/01. eng., 86(8), 2708-12.

[13] Vinken, M, Vanhaecke, T, Papeleu, P, Snykers, S, Henkens, T, \& Rogiers, V. Connexins and their channels in cell growth and cell death. Cell Signal. (2006). May;PubMed PMID: 16183253. Epub 2005/09/27. eng., 18(5), 592-600. 
[14] Laird, D. W. Life cycle of connexins in health and disease. The Biochemical journal. (2006). Mar 15;PubMed PMID: PMID: 16492141; BJ20051922 [pii]., 394(3), 527-43.

[15] Sohl, G, Degen, J, Teubner, B, \& Willecke, K. The murine gap junction gene connexin36 is highly expressed in mouse retina and regulated during brain development. FEBS Letters. (1998). May 22;428(1-2):27-31. PubMed PMID: 9645468. Epub 1998/06/30. eng.

[16] Condorelli, D. F, Parenti, R, \& Spinella, F. Trovato Salinaro A, Belluardo N, Cardile $\mathrm{V}$, et al. Cloning of a new gap junction gene (Cx36) highly expressed in mammalian brain neurons. European Journal of Neuroscience. (1998). Mar;PubMed PMID: 9753189. Epub 1998/09/30. eng., 10(3), 1202-8.

[17] Serre-beinier, V, Bosco, D, Zulianello, L, Charollais, A, Caille, D, Charpantier, E, et al. Cx36 makes channels coupling human pancreatic beta-cells, and correlates with insulin expression. Human molecular genetics. (2009). Feb 1;PubMed PMID: 19000992. Epub 2008/11/13. eng., 18(3), 428-39.

[18] Belluardo, N, Mudo, G, \& Trovato-salinaro, A. Le Gurun S, Charollais A, Serre-Beinier $\mathrm{V}$, et al. Expression of connexin36 in the adult and developing rat brain. Brain Research. (2000). May 19;PubMed PMID: 10814742. Epub 2000/05/18. eng., 865(1), 121-38.

[19] Gulisano, M, Parenti, R, Spinella, F, \& Cicirata, F. Cx36 is dynamically expressed during early development of mouse brain and nervous system. Neuroreport. (2000). Nov 27;PubMed PMID: 11117498. Epub 2000/12/16. eng., 11(17), 3823-8.

[20] Connors, B. W, \& Long, M. A. Electrical synapses in the mammalian brain. Annual Review of Neuroscience. (2004). PubMed PMID: PMID: 15217338., 27, 393-418.

[21] Bertram, J. S, \& Vine, A. L. Cancer prevention by retinoids and carotenoids: independent action on a common target. Biochimica et biophysica acta. (2005). May 30;PubMed PMID: PMID: 15949684; S0925-4439(05)00003-7 [pii]., 1740(2), 170-8.

[22] Stahl, W, \& Sies, H. The role of carotenoids and retinoids in gap junctional communication. International journal for vitamin and nutrition research. (1998). PubMed PMID: 9857261. Epub 1998/12/19. eng., 68(6), 354-9.

[23] Vine, A. L, \& Bertram, J. S. Upregulation of connexin 43 by retinoids but not by nonprovitamin A carotenoids requires RARs. Nutrition and cancer. (2005). PubMed PMID: PMID: 16091010., 52(1), 105-13.

[24] Cicirata, F, Parenti, R, Spinella, F, Giglio, S, Tuorto, F, Zuffardi, O, et al. Genomic organization and chromosomal localization of the mouse Connexin36 (mCx36) gene. Gene. (2000). Jun 27;PubMed PMID: 10876089. Epub 2000/07/06. eng., 251(2), 123-30.

[25] Encinas, M, Iglesias, M, Liu, Y, Wang, H, Muhaisen, A, Cena, V, et al. Sequential treatment of SH-SY5Y cells with retinoic acid and brain-derived neurotrophic factor gives rise to fully differentiated, neurotrophic factor-dependent, human neuron-like 
cells. Journal of Neurochemistry. (2000). Sep;PubMed PMID: 10936180. Epub 2000/08/11. eng., 75(3), 991-1003.

[26] Agholme, L, Lindstrom, T, Kagedal, K, Marcusson, J, \& Hallbeck, M. An in vitro model for neuroscience: differentiation of SH-SY5Y cells into cells with morphological and biochemical characteristics of mature neurons. J Alzheimers Dis. (2010). PubMed PMID: 20413890. Epub 2010/04/24. eng., 20(4), 1069-82.

[27] Abramoff, M. D, Magelhaes, P. J, \& Ram, S. J. Image Processing with ImageJ. Biophotonics International. (2004). , 11(7), 36-42.

[28] Meijering, E, Jacob, M, Sarria, J. C, Steiner, P, Hirling, H, \& Unser, M. Design and validation of a tool for neurite tracing and analysis in fluorescence microscopy images. Cytometry A. (2004). Apr;PubMed PMID: 15057970. Epub 2004/04/02. eng., 58(2), 167-76.

[29] Clagett-dame, M, Mcneill, E. M, \& Muley, P. D. Role of all-trans retinoic acid in neurite outgrowth and axonal elongation. Journal of neurobiology. (2006). Jun;PubMed PMID: PMID: 16688769., 66(7), 739-56.

[30] Larsson, C. Protein kinase $C$ and the regulation of the actin cytoskeleton. Cell Signal. (2006). Mar;PubMed PMID: 16109477. Epub 2005/08/20. eng., 18(3), 276-84.

[31] Edsjo, A, Lavenius, E, Nilsson, H, Hoehner, J. C, Simonsson, P, Culp, L. A, et al. Expression of trkB in human neuroblastoma in relation to MYCN expression and retinoic acid treatment. Laboratory investigation; a journal of technical methods and pathology. (2003). Jun;PubMed PMID: PMID: 12808116., 83(6), 813-23.

[32] Hokfelt, T, Stanic, D, Sanford, S. D, Gatlin, J. C, Nilsson, I, Paratcha, G, et al. NPY and its involvement in axon guidance, neurogenesis, and feeding. Nutrition. (2008). Sep;PubMed PMID: PMID: 18725084; S0899-9007(08)00276-1 [pii]., 24(9), 860-8.

[33] Reynolds, C. P, Matthay, K. K, Villablanca, J. G, \& Maurer, B. J. Retinoid therapy of high-risk neuroblastoma. Cancer letters. (2003). Jul 18;197(1-2):185-92. PubMed PMID: PMID: 12880980; S0304383503001083 [pii].

[34] Haussler, M, Sidell, N, Kelly, M, Donaldson, C, Altman, A, \& Mangelsdorf, D. Specific high-affinity binding and biologic action of retinoic acid in human neuroblastoma cell lines. Proceedings of the National Academy of Sciences of the United States of America. (1983). Sep;PubMed PMID: 6310582. Pubmed Central PMCID: PMC384290. Epub 1983/09/01. eng., 80(18), 5525-9.

[35] Kelsey, L, Katoch, P, Johnson, K. E, Batra, S. K, \& Mehta, P. P. Retinoids regulate the formation and degradation of gap junctions in androgen-responsive human prostate cancer cells. PloS one. (2012). e32846. PubMed PMID: 22514600. Pubmed Central PMCID: PMC3326013. Epub 2012/04/20. eng.

[36] Rudkin, G. H, Carlsen, B. T, Chung, C. Y, Huang, W, Ishida, K, Anvar, B, et al. Retinoids inhibit squamous cell carcinoma growth and intercellular communication. The 
Journal of surgical research. (2002). Apr;PubMed PMID: 11922733. Epub 2002/04/02. eng., 103(2), 183-9.

[37] Hartfield, E. M, Rinaldi, F, Glover, C. P, Wong, L. F, Caldwell, M. A, \& Uney, J. B. Connexin 36 expression regulates neuronal differentiation from neural progenitor cells. PloS one. (2011). e14746. PubMed PMID: 21408068. Epub 2011/03/17. eng.

[38] Todorova, M. G, Soria, B, \& Quesada, I. Gap junctional intercellular communication is required to maintain embryonic stem cells in a non-differentiated and proliferative state. Journal of cellular physiology. (2008). Feb;PubMed PMID: PMID: 17654515., 214(2), 354-62.

[39] Mclachlan, E, Shao, Q, \& Laird, D. W. Connexins and gap junctions in mammary gland development and breast cancer progression. The Journal of membrane biology. (2007). Aug;218(1-3):107-21. PubMed PMID: 17661126. Epub 2007/07/31. eng.

[40] Lee, S. C, Cruikshank, S. J, \& Connors, B. W. Electrical and chemical synapses between relay neurons in developing thalamus. Journal of Physiology. (2010). Jul 1;588(Pt 13):2403-15. PubMed PMID: 20457735. Epub 2010/05/12. eng.

[41] Bez, A, Corsini, E, Curti, D, Biggiogera, M, Colombo, A, Nicosia, R. F, et al. Neurosphere and neurosphere-forming cells: morphological and ultrastructural characterization. Brain Research. (2003). Dec 12;993(1-2):18-29. PubMed PMID: 14642827. Epub 2003/12/04. eng.

[42] Ross, R. A, Spengler, B. A, \& Biedler, J. L. Coordinate morphological and biochemical interconversion of human neuroblastoma cells. Journal of the National Cancer Institute. (1983). Oct;PubMed PMID: 6137586. Epub 1983/10/01. eng., 71(4), 741-7.

[43] Wei, C. J, Francis, R, Xu, X, \& Lo, C. W. Connexin43 associated with an N-cadherincontaining multiprotein complex is required for gap junction formation in NIH3T3 cells. Journal of Biological Chemistry. (2005). May 20;PubMed PMID: 15741167. Epub 2005/03/03. eng., 280(20), 19925-36.

[44] Jensen, M, \& Berthold, F. Targeting the neural cell adhesion molecule in cancer. Cancer Letters. (2007). Dec 8;PubMed PMID: 17949897. Epub 2007/10/24. eng., 258(1), 9-21.

[45] Nishimura, T, Dunk, C, Lu, Y, Feng, X, Gellhaus, A, Winterhager, E, et al. Gap junctions are required for trophoblast proliferation in early human placental development. Placenta. (2004). Aug;PubMed PMID: 15193866. Epub 2004/06/15. eng., 25(7), 595-607.

[46] Striedinger, K, Petrasch-parwez, E, Zoidl, G, Napirei, M, Meier, C, Eysel, U. T, et al. Loss of connexin36 increases retinal cell vulnerability to secondary cell loss. European Journal of Neuroscience. (2005). Aug;PubMed PMID: 16101742. Epub 2005/08/17. eng., 22(3), 605-16.

[47] Gonzalez-nieto, D, Gomez-hernandez, J. M, Larrosa, B, Gutierrez, C, Munoz, M. D, Fasciani, I, et al. Regulation of neuronal connexin-36 channels by $\mathrm{pH}$. Proceedings of 
the National Academy of Sciences of the United States of America. (2008). Nov 4;PubMed PMID: PMID: 18957549; 0804189105 [pii]., 105(44), 17169-74.

[48] Srinivas, M, Rozental, R, Kojima, T, Dermietzel, R, Mehler, M, Condorelli, D. F, et al. Functional properties of channels formed by the neuronal gap junction protein connexin36. Journal of Neuroscience. (1999). Nov 15;PubMed PMID: 10559394. Epub 1999/11/13. eng., 19(22), 9848-55.

[49] Oguro, K, Jover, T, Tanaka, H, Lin, Y, Kojima, T, Oguro, N, et al. Global ischemiainduced increases in the gap junctional proteins connexin 32 (Cx32) and Cx36 in hippocampus and enhanced vulnerability of Cx32 knock-out mice. Journal of Neuroscience. (2001). Oct 1;PubMed PMID: 11567043. Epub 2001/09/22. eng., 21(19), 7534-42.

[50] Voigt, A, \& Zintl, F. Effects of retinoic acid on proliferation, apoptosis, cytotoxicity, migration, and invasion of neuroblastoma cells. Medical and pediatric oncology. (2003). Apr;PubMed PMID: 12555246. Epub 2003/01/30. eng., 40(4), 205-13.

[51] Lofstedt, T, Jogi, A, Sigvardsson, M, Gradin, K, Poellinger, L, Pahlman, S, et al. Induction of ID2 expression by hypoxia-inducible factor-1: a role in dedifferentiation of hypoxic neuroblastoma cells. Journal of Biological Chemistry. (2004). Sep 17;PubMed PMID: 15252039. Epub 2004/07/15. eng., 279(38), 39223-31.

[52] Wiese, C, Rolletschek, A, Kania, G, Blyszczuk, P, Tarasov, K. V, Tarasova, Y, et al. Nestin expression--a property of multi-lineage progenitor cells? Cellular and molecular life sciences. (2004). Oct;61(19-20):2510-22. PubMed PMID: 15526158. Epub 2004/11/05. eng. 